\title{
Situación y desafíos del transporte público de pasajeros en la ciudad de Huaraz
}

Situation and challenges of public transport of passengers in the city of Huaraz

$$
\text { JoHn TARAZONA JiMÉNEZ }{ }^{1}
$$

\section{RESUMEN}

Analiza la situación del transporte público en la ciudad de Huaraz, departamento de Áncash, Perú. Para ello se ha tomado en cuenta las características de la oferta de transporte público y de la demanda. El análisis de la demanda centrada en hogares que no disponen de vehículos propios ha permitido conocer que el $75.92 \%$ de la población urbana no cuenta con vehículo propio, por lo que podemos afirmar que de cada diez habitantes, ocho no cuentan con ningún tipo de movilidad por lo que son usuarios permanentes del transporte público en sus diferentes modalidades. El análisis de la encuesta de origen y destino, permitió identificar a las zonas con mayor afluencia de pasajeros en los distritos de Huaraz e Independencia. Entre estas podemos mencionar a la Av. Raymondi, Av. Fitzcarrald, y los jirones Huascarán, Caraz, San Cristóbal. En segundo lugar, se han identificado a la avenida Luzuriaga, jirones San Martín, Simón Bolívar, Av. 28 de Julio y el Jr. José de Sucre. En el distrito de Independencia la zona de mayor afluencia de pasajeros comprende a las avenidas Universitaria y Eucaliptos, y los jirones César Vallejo, Membrillos, Palmeras y Quisuares (Shancayán); en segundo lugar, la zona que comprende la Av. Independencia, Av. Centenario, los pasajes Sarita Colonia, calle Santa Rosa y cruce Palmira. El estudio permite demostrar las horas de mayor afluencia (horas punta); por la mañana, la hora punta comprende de 7 a 8; luego, al mediodía, de 12:40 a la 13:30; por las tardes, de 18:00 a 18:30. Así mismo, la investigación ha revelado que de cada 100 vehículos que se desplazan en las horas punta, 79 son autos colectivos, 15 camionetas rurales y 6 vehículos particulares. Además, muestra que en la ciudad de Huaraz existe una sobre oferta del servicio de transporte público durante gran parte del

1 Universidad Nacional Santiago Antúnez de Mayolo. Huaraz, Perú. 
día en la modalidad de servicio de taxi colectivo, generando externalidades negativas, como la congestión vehicular, mayor contaminación y ruido en la ciudad.

Palabras clave: transporte público; hora punta; encuesta de origen y destino; congestión vehicular.

\section{ABSTRACT}

The aim of the following research is to analyze the situation of the public transport in the city of Huaraz, department of Áncash, Peru, for this, the features of the supply of public transport and the features of the demand have been taken. The analysis of the demand, focused at homes with no own vehicles, has let us know that a 75.92 percent of the city people in Huaraz don't own vehicles, so we can say for truth that for every ten citizens in the city of Huaraz, eight of them don't have any kind of means of transport, just being permanent users of public transport on its different ways. The analysis of the destiny and origin survey was able to identify over the areas with great influence of passenger in the district of Huaraz and Independencia, this makes us mention some avenues such as: Raymondi. Av. Fitzcarrald, Av. and the streets Caraz, San Cristobal and in second place, the Luzuriaga Avenue, the streets San Martin, Simon Bolivar, 28 de Julio Avenue and Jose de Sucre Street have been identified. In the district of Independencia the area with great influence of passengers covers the Universitaria, Eucaliptos avenues and the streests Cesar Vallejo, Membrillos, Palmeras, Quisuares (Shancayan) and in second place the area that covers the avenues Independencia, Centenario and the alleyways Sarita Colonia, Santa Rosa street and crossing Palmira. The study has identified that the area with great influence (rush hour) is given in the morning, the rush hours is from seven to eight a.m. then this phenomenon keeps on from twelve- forty to half past one p.m., in the evenings from six to half past six, furthermore the study has found a reward that in stitching with great influence of congestion during rush hour are the collective cars which ones cause the greatest car congestion, for each hundred vehicles driving during the rush hour sixty-nine of them are collective cars, fifteen of them are rural pickups and six of them are private cars. Furthermore the study reveals that in Huaraz city there is an oversupply of service of public transport during the most time during the day, and they are mainly taxi cabs and collectives generating a negative impact such as: car congestion, pollution and noise in the city.

Keywords: public transport; rush hour; origin and destination survey; congestion. 


\section{INTRODUCCIÓN}

Entre los temas que afectan el desarrollo de ciudades grandes, pequeñas e intermedias se puede señalar a la seguridad ciudadana, el comercio informal y el transporte. Este trabajo pretende iniciar la discusión sobre el problema de transporte, y en particular sobre el transporte público de pasajeros. Siendo el objetivo de la investigación describir y analizar la situación del transporte público en la ciudad de Huaraz, departamento de Áncash, Perú.

La ciudad de Huaraz tiene un crecimiento desordenado del parque automotor, fenómeno propio de las grandes ciudades y ciudades intermedias, sin embargo el exceso de la oferta de servicios de transporte público de pasajeros en unidades más pequeñas desde mototaxis, taxis colectivos y camionetas rurales ha generado que en las horas punta la aglomeración y saturación de vías; reduzca el tránsito, causando malestar en los usuarios y proveedores del servicio de transporte. Los gobiernos locales tienen la competencia en materia de tránsito, circulación y transporte público dentro de la provincia, por lo que autoriza, supervisa y fiscaliza la prestación de servicios de transporte dentro del ámbito provincial; sin embargo no vienen atendiendo sus funciones y competencia de manera técnica, por lo que el problema sigue agudizándose.

Es necesario recalcar que "la planeación del transporte pretende dar óptimo uso a la infraestructura vial y medios de transporte, de tal forma que se atienda las necesidades de movilidad de la población" (Posada et. al., 2010), por lo que la administración municipal debería evaluar y analizar los resultados del estudio que se presentan en este artículo, para tomar acciones correctivas.

\section{MATERIALES Y MÉTODOS}

El tipo de investigación fue descriptiva, de corte transversal, la población de estudio se encuentra constituida por personas entre los 18 a 70 años de la ciudad de Huaraz con un total de 73 408. La muestra empleada para el estudio está formada por 245 personas. La fórmula aplicada fue la siguiente:

$$
\mathrm{n}=\frac{z^{2} * p^{*} q^{*} N}{E^{2} *(N-1)+Z^{2} * P * Q}
$$

Así mismo se empleó el estudio de ascensos y descensos de pasajeros en las zonas de mayor concurrencia, a través de fichas de observación. 


\section{RESULTADOS}

La disponibilidad de vehículos en los hogares entrevistados nos permitió evaluar la demanda del transporte público de pasajeros. Los resultados obtenidos nos muestran que el 76\% de los hogares no tienen vehículos, el 24\% sí tiene al menos uno.

Tabla 1. Vehículos disponibles en el hogar, 2014

\begin{tabular}{ccc}
\hline Vehículos Disponibles & Frecuencia & $\%$ \\
\hline Total & 245 & $100.00 \%$ \\
No tienen Vehículo & 186 & $75.92 \%$ \\
Sí tienen Vehículo & 59 & $24.08 \%$ \\
Con 1 Vehículo & 51 & $20.82 \%$ \\
Con 2 Vehículos & 6 & $2.45 \%$ \\
Con 3 Vehículos & 2 & $0.82 \%$ \\
\hline
\end{tabular}

El análisis de las zonas y manzanas de mayor afluencia nos permite conocer el destino de las personas dentro de la ciudad de Huaraz, para ello se ha utilizado mapas especialmente diseñados y validados por el Instituto Nacional de Estadística e Informática - INEI, donde se han fijado las zonas y manzanas que se utilizó para identificar nuestras Zonas de análisis de Tráfico (ZAT) tomando la densidad del viaje (número de viajes dividido por el área de la zona).

En el distrito de Huaraz se han conformado 9 zonas (manzanas y calles) de acuerdo a la encuesta de origen y destino. Aquellas de mayor demanda son la zona 1 que comprende las avenidas Raymondi, Fitzcarrald, y los jirones Huascarán, Caraz, San Cristóbal; en segundo lugar se identificó la zona 4 que comprende a la avenida Luzuriaga, jirones San Martín, Simón Bolívar, Av. 28 de Julio y el Jr. José de Sucre. 


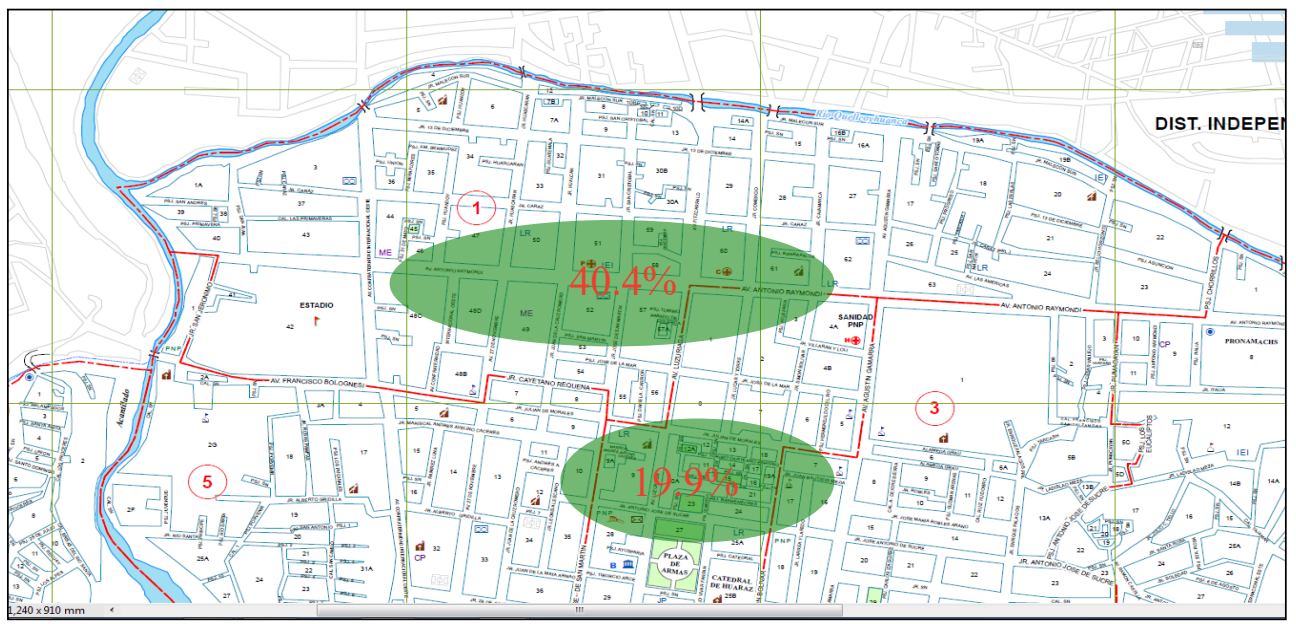

1. Zona de mayor afluencia de pasajeros - Huaraz

Para el caso del distrito de Independencia se determinó que las zonas con mayor afluencia son las avenidas Universitaria, Av. Eucaliptos y jirones César Vallejo, Jr. Membrillos, Palmeras, Quisuares; y en segundo lugar, la zona que comprende la Av. Independencia, Av. Centenario, los pasajes Sarita Colonia, Calle Santa Rosa y cruce Palmira.

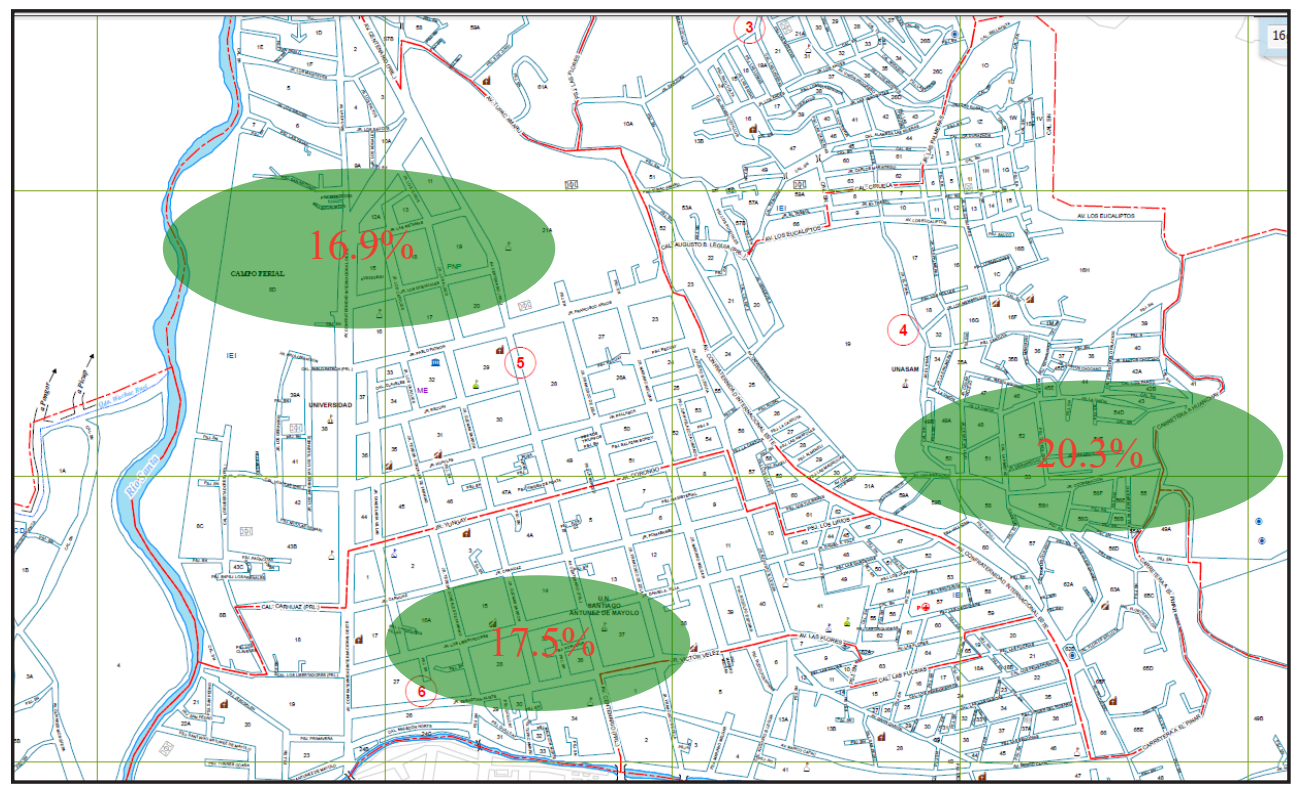

Figura 2. Zona de mayor afluencia de pasajeros - Independencia

La información de la afluencia de pasajeros y la evaluación se ascensos y descensos de pasajeros a través de las fichas de aforo, nos ha permitido identificar las vías de mayor tránsito y saturación que se ubican en las siguientes avenidas y jirones: 
Tabla 2. Vías de mayor tránsito

\begin{tabular}{|c|c|c|c|}
\hline Punto & Vía & $\begin{array}{l}\text { Ubicación } \\
\text { distrital }\end{array}$ & Nodos conflictivos \\
\hline 1 & Jr. San Martín & Huaraz & $\begin{array}{c}\text { Intersección del Jr. } 28 \text { de Julio con el Jr. } \\
\text { San Martín }\end{array}$ \\
\hline 2 & Jr. Simón Bolívar & Huaraz & $\begin{array}{l}\text { Intersección del Jr. Simón Bolívar con el } \\
\text { Jr. Julián de Morales. }\end{array}$ \\
\hline 3 & $\begin{array}{l}\text { Av. } 27 \text { de Noviembre } \\
\text { (Ex - Tarapacá) }\end{array}$ & Huaraz & $\begin{array}{l}\text { Intersección de la Av. } 27 \text { de Noviembre } \\
\text { con el Jr. Cayetano Requena }\end{array}$ \\
\hline 4 & Av. Centenario & Independencia & $\begin{array}{c}\text { Av. Centenario, intersección con la } \\
\text { UNASAM }\end{array}$ \\
\hline 5 & Av. Raymondi & Huaraz & $\begin{array}{l}\text { Intersección de la Av. Raymondi con el Jr. } \\
\text { Juan de la Cruz Romero }\end{array}$ \\
\hline 6 & Av. Fitzcarrald & Huaraz & $\begin{array}{c}\text { Intersección de la Av. Fitzcarrald con el Jr. } \\
\text { Caraz }\end{array}$ \\
\hline 7 & $\begin{array}{l}\text { Av. Confraternidad } \\
\text { Internacional Oeste }\end{array}$ & Huaraz & $\begin{array}{l}\text { Intersección de la Av. Confraternidad } \\
\text { Internacional Oeste con la Av. Raymondi }\end{array}$ \\
\hline 8 & Jr. San Martin & Huaraz & $\begin{array}{c}\text { Intersección del Jr. San Martín con el Jr. } \\
\text { José de Sucre }\end{array}$ \\
\hline 9 & $\begin{array}{l}\text { Av. Francisco } \\
\text { Bolognesi }\end{array}$ & Huaraz & $\begin{array}{c}\text { Cruce con la Av. Confraternidad } \\
\text { Internacional Oeste. Av. } 27 \text { de Noviembre } \\
\text { (Ex - Tarapacá) }\end{array}$ \\
\hline
\end{tabular}

En los nueve puntos de mayor tránsito existe superposición en el flujo de transporte, aquí transitan vehículos particulares, autos colectivos, taxis, transporte público urbano e interurbano, incluso vehículos de carga y buses interprovinciales.

Si evaluamos los promedios en las vías de mayor tránsito, por cada cien vehículos que transitan en hora punta 79 son autos colectivos, 15 camionetas rurales y 6 vehículos particulares.

La cantidad de pasajeros transportados en promedio al día llega a los 277 260, lo que muestra una gran movilidad interna, por actividades como las compras diarias, trámites en instituciones públicas y privadas, viajes a centros laborales y de estudio, viajes que se hacen con mucha frecuencia debido a la cercanía y el tiempo de viaje, propio de una ciudad pequeña y centralizada, lo que demanda un flujo mayor de vehículos, generándose problemas de congestionamiento.

En la mañana y al medio día se registra la mayor afluencia de pasajeros en comparación con la tarde; sin embargo se requiere investigaciones de mayor profundidad para explicar aquellas preferencias que son dependientes del tiempo y que determinan el proceso de movilización y uso de medios de transporte. Las razones de este comportamiento pueden ser múltiples y varían según restric- 
ciones (horarios de ingreso y salida), normas (número de horas día, modalidad de contrato), incluso contingencias (condiciones meteorológicas), así como la combinación de tales efectos (Märki et al., 2014).

\section{DISCUSIÓN}

El transporte y su problemática no han sido abordados de manera adecuada por las autoridades locales de la Municipalidad Provincial de Huaraz. El desafío hacia el futuro es mayor si tomamos en cuenta el rol que asume el transporte en el desarrollo de la ciudad como es: propiciar canales de comunicación y articulación entre las distintas centralidades, así como entre estas y su tejido residencial a su alrededor (Aparicio, 2010).

El proceso de ordenamiento del territorio debe ser coherente con la planificación del transporte. Su importancia dentro del proceso del ordenamiento radica en la influencia que ejerce el transporte en la ciudad en los procesos de expansión territorial y la localización de los usos. Es decir, existe una estrecha relación entre los modelos territoriales urbanos y los esquemas de movilidad (García, 2013).

En el caso del distrito de Huaraz se ha separado en 09 puntos de análisis, ubicándose como la de mayor concurrencia la zona 1 que comprende la Av. Antonio Raymondi con las intersecciones del Jr. Juan de la Cruz Romero y el Jr. 27 de noviembre. Esta alta concurrencia se explicaría por encontrarse en esta zona el mercado central, así como la parada Quillcay y otros lugares concurridos para la compra de productos de primera necesidad.

Otro punto de alta concurrencia de pasajeros corresponde a la zona 4, Av. Luzuriaga con el Jr. José de Sucre, en donde se ubican instituciones públicas y bancarias. Se puede identificar en esta zona a la Municipalidad Provincial de Huaraz, el Poder Judicial, Ministerio Público, la Comisaría de la Policía Nacional, el Banco de la Nación, El Instituto Nacional de Cultura, el local de los bomberos, el Centro Cultural, y diferentes instituciones financieras; factores que explicarían la demanda de transporte en la zona de referencia.

En el distrito de Independencia podemos indicar que la zona de mayor concurrencia es la zona 4, que corresponde a la Av. Centenario con el Jr. Víctor Vélez, ahí se encuentra el local Central de la Universidad Nacional Santiago Antúnez de Mayolo (UNASAM), además del Colegio Nacional Antonio Raymondi. Este punto además sirve como paradero y nodo a diferentes zonas del distrito, conectando las zonas de Nicrupampa, Shancayán y el Pinar para el Este, o para el Norte en dirección a las zonas de Cascapampa, Vichay, El Milagro, Palmira. 
El otro punto con mayor concurrencia es la ciudad universitaria de la Universidad Nacional Santiago Antúnez de Mayolo - UNASAM, que se encuentra en la zona de Shancayán, zona 1. La Av. Centenario con el Jr. Pablo Patrón es también una de las zonas con mayor concurrencia. Aquí se encuentran instituciones públicas como la Municipalidad Distrital de Independencia, la Institución Educativa GUE Toribio de Luzuriaga, la SUNARP, entre otras.

Se puede contribuir a la solución del problema del transporte en la ciudad de Huaraz si se reduce la cantidad de vehículos de transporte público que circulan, y esto implicaría reemplazar los vehículos que brindan el servicio de taxis-colectivos por unidades de mayor capacidad como las camionetas rurales. El cálculo de corto plazo demuestra que la flota total para atender la demanda diaria sería de 556 con una frecuencia media de 1.2 minutos. Actualmente las 12 empresas de transportes analizadas cuentan con 414 unidades con una frecuencia promedio de 3 minutos en las horas punta. Cabe recordar que la gestión orientada a la calidad en las municipalidades se orienta hoy a controlar el proceso tecnológico de los servicios de transporte público con el objetivo de reducir los factores que amenazan la seguridad vial y la salud (Boyko, Tujrin y Fedotov, 2015).

La propuesta de solución al problema del trasporte público en la ciudad de Huaraz incluye contemplar como una opción en el mediano plazo el reemplazo de vehículos menores y livianos por vehículos con mayor capacidad (30 asientos). En el escenario actual se requieren 296 unidades con frecuencias de salidas de cada 3 minutos. Esto demuestra que las mejoras en los servicios de transporte público a través de la planificación es un componente importante en el desarrollo de las regiones, contar con servicios de calidad proporciona diversos beneficios económicos, sociales y ambientales (Litman, 2014).

\section{CONCLUSIONES}

La sobreposición en el uso de las principales vías por el tránsito de vehículos particulares, taxi colectivos, camionetas rurales, incluso vehículos pesados y mototaxis por vías principales; agudiza el caos y desorden en el transporte. Esta situación es explicada principalmente por la capacidad de oferta de los servicios de transporte en vehículos tipo taxi y colectivo. En la investigación se ha identificado a 25 empresas con 1653 vehículos autorizados por la Municipalidad Provincial de Huaraz, además existe un número importante de vehículos que prestan el servicio de taxi colectivo de manera informal; por lo que el sistema de movilidad urbana de la Ciudad de Huaraz se basa simplemente en automóviles que realizan el servicio de taxi colectivo (que constituyen el 79\% del total de vehículos que transitan en las zonas de mayor flujo vehicular). 
El diseño de los instrumentos de planificación territorial y del transporte son tareas pendientes que requieren su atención prioritaria. Para ello se debe sustituir el uso de vehículos tipo taxi colectivo por vehículos de mayor aforo, medida que debe ir acompañada con la restricción en la circulación de vehículos particulares en las vías de mayor saturación. Es posible con la flota existente de camionetas rurales, satisfacer gran parte de la demanda actual, reduciendo las frecuencias de salida por lo que esta medida no debiera afectar a los usuarios del transporte público.

Es necesario evaluar la dinámica social y económica así como los efectos que se generan en el tránsito y el transporte. La encuesta de origen y destino cumple ese rol y permite observar parte de las dinámicas territoriales. En consecuencia, la concesión de nuevas rutas y las autorizaciones del tamaño de flota en el transporte público debe acompañarse con un estudio técnico no menor a una vez al año.

\section{AGRADECIMIENTOS}

A la Universidad Nacional Santiago Antúnez de Mayolo, por la oportunidad de realizar actividades académicas y de investigación.

\section{REFERENCIA BIBLIOGRÁFICA}

Agencia de Cooperación Internacional del Japón (JICA). «Encuesta de recolección de información básica del transporte urbano en el área metropolitana de Lima y Callao». Informe Final, 2013 (Lima: Agencia de Cooperación Internacional del Japón, 2013), 61.

Aparicio, Liliana. 2010. «El impacto del transporte en el ordenamiento de la ciudad: el caso de Transmilenio en Bogotá». Revista De Estudios Regionales Y Urbanos, N²2. Bogotá: Universidad del Rosario. 33-64.

Boyko, Grigory y otros. 2015. «Research of Influence of Small Capacity Passenger». transport problems. Vol. 10, No 1. Poland: Faculty of Transport, The Silesian University of Technology. 23-30.

García Schilardi y María Emilia. 2013. «Organización Territorial Urbana Y Transporte Público Masivo: Metrópolis Del Gran Mendoza». Urbano, Vol. 16, N²8. Universidad del Bio - Bio. 50-57.

Litman, Todd. 2014. «Evaluating Public Transportation». Journal of Public Transportation, Vol 17, $\mathrm{N}^{\circ}$ 1. University of South Florida. 43-74. 
Märki, Fabian y otros. 2014. «Location choice for a continuous simulation of long periods under changing conditions». Journal of Transport and Land Use, Vol 7, No 2. University of Minnesota. 1-17.

Posada Henao y otros 2010. «Metodología para estudio de demanda de transporte público de pasajeros en zonas rurales». Revista Facultad de Ingeniería Universidad de Antioquia. N 53. Medellin. Universidad de Antioquia. 106118.

Presentado: $20 / 01 / 2017$

Aceptado: $21 / 04 / 2017$

\section{Correspondencia}

John Joseph Tarazona Jiménez johntarazona@gmail.com 